\title{
Numerical Radius Inequalities for Sums and Products of Operators
}

\author{
Wasim Audeh \\ Department of Mathematics, Petra University, Amman, Jordan \\ Email: waudeh@uop.edu.jo.
}

How to cite this paper: Audeh, W. (2019) Numerical Radius Inequalities for Sums and Products of Operators. Advances in Linear Algebra \& Matrix Theory, 9, 35-42. https://doi.org/10.4236/alamt.2019.93003

Received: June 13, 2019

Accepted: July 7, 2019

Published: July 10, 2019

Copyright (C) 2019 by author(s) and Scientific Research Publishing Inc. This work is licensed under the Creative Commons Attribution International License (CC BY 4.0).

http://creativecommons.org/licenses/by/4.0/

\section{Abstract}

A numerical radius inequality due to Shebrawi and Albadawi says that: If $A_{i}, B_{i}, X_{i}$ are bounded operators in Hilbert space, $i=1,2, \cdots, n$, and $f, g$ be nonnegative continuous functions on $[0, \infty)$ satisfying the relation $f(t) g(t)=t \quad(t \in[0, \infty))$, then

$w^{r}\left(\sum_{i=1}^{n} A_{i}^{*} X_{i} B_{i}\right) \leq \frac{n^{r-1}}{2}\left\|\sum_{i=1}^{n}\left(\left[A_{i}^{*} g^{2}\left(\left|X_{i}^{*}\right|\right) A_{i}\right]^{r}+\left[B_{i}^{*} f^{2}\left(\left|X_{i}\right|\right) B_{i}\right]^{r}\right)\right\|$ for all

$r \geq 1$. We give sharper numerical radius inequality which states that: If $A_{i}, B_{i}, X_{i}$ are bounded operators in Hilbert space, $i=1,2, \cdots, n$, and $f, g$ be nonnegative continuous functions on $[0, \infty)$ satisfying the relation

$f(t) g(t)=t \quad(t \in[0, \infty))$, then

$$
\begin{aligned}
& w^{r}\left(\sum_{i=1}^{n} A_{i}^{*} X_{i} B_{i}\right) \leq \frac{n^{r-1}}{2}\left\|\sum_{i=1}^{n}\left[A_{i}^{*} g^{2}\left(\left|X_{i}^{*}\right|\right) A_{i}\right]^{r}+\left[B_{i}^{*} f^{2}\left(\left|X_{i}\right|\right) B_{i}\right]^{r}\right\|-\alpha \text { where } \\
& \alpha=\sup _{\|x\|=1} \frac{n^{r-1}}{2} \sum_{i=1}^{n}\left(\left\langle\left[A_{i}^{*} g^{2}\left(\left|X_{i}^{*}\right|\right) A_{i}\right]^{r} x, x\right\rangle^{1 / 2}-\left\langle\left[B_{i}^{*} f^{2}\left(\left|X_{i}\right|\right) B_{i}\right]^{r} x, x\right\rangle^{1 / 2}\right)^{2} .
\end{aligned}
$$

Moreover, we give many numerical radius inequalities which are sharper than related inequalities proved recently, and several applications are given.

\section{Keywords}

Numeriacl Radius, Operator Norm, Operator Matrix, Inequality, Equality, Offdiagonal Part

\section{Fundamental Principles}

Let $B(H)$ denote the $C^{*}$-algebra of all bounded linear operators on a Hilbert space $H$. In the case when $\operatorname{dim} H=n$, we identify $B(H)$ with the matrix algebra $M_{n}$ of all $n \times n$ matrices with entries in the complex field. The numeri- 
cal radius of $T \in B(H)$ is defined by

$$
w(T)=\sup \{|\langle T x, x\rangle|: x \in H,\|x\|=1\} .
$$

It is well-known that $w($.$) defines a norm on B(H)$, which is equivalent to the usual operator norm. Namely, for $T \in B(H)$, we have

$$
\frac{\|T\|}{2} \leq w(T) \leq\|T\|
$$

These inequalities are sharp. The first inequality becomes an equality if $T^{2}=0$, and the second inequality becomes an equality if $T$ is normal (see [1]).

An important inequality for $w(T)$ is the power inequality stating that $w\left(X^{n}\right) \leq(w(X))^{n}$ for $n=1,2, \cdots$ see ([2]: p. 118).

An important property of the numerical radius norm is its weak unitary invariance, that is, for $X \in B(H)$,

$$
w\left(U^{*} X U\right)=w(X)
$$

for every unitary $U \in B(H)$. For further information about the properties of numerical radius inequalities we refer the reader to [2]-[7] and references therein.

Let $H_{1}, H_{2}$ be Hilbert spaces, and consider the direct sum $H=H_{1} \oplus H_{2}$. By considering this decomposition, every operator $T \in B(H)$ has a $2 \times 2$ operator matrix representation $T=\left[T_{i j}\right]$ with entries $T_{i j} \in B\left(H_{1} \oplus H_{2}\right)$.

\section{Introduction}

Hirzallah, Kittaneh and Shebrawi have proved in [8] that:

If $X \in B(H)$, then:

$$
\frac{\|X\|}{2}+\frac{\|\operatorname{Re} X\|-\|\operatorname{Im} X\|}{2} \leq w(X)
$$

also, they proved that:

If $X \in B(H)$, then:

$$
\frac{\|X\|}{2}+\frac{\left|\|\operatorname{Re} X\|-\frac{\|X\|}{2}\right|}{4}+\frac{\left|\|\operatorname{Im} X\|-\frac{\|X\|}{2}\right|}{4} \leq w(X)
$$

Moreover, they showed that:

if $X, Y \in B(H)$, then:

$$
w\left(\left[\begin{array}{cc}
0 & X \\
Y & 0
\end{array}\right]\right) \leq \frac{w(X+Y)+w(X-Y)}{2}
$$

Shebrawi and Albadawi have proved in [9] that:

If $A_{i}, B_{i}, X_{i} \in B(H),(i=1,2, \cdots, n)$ and $f, g$ be nonnegative continuous functions on $[0, \infty)$ satisfying the relation $f(t) g(t)=t \quad(t \in[0, \infty))$, then:

$$
w^{r}\left(\sum_{i=1}^{n} A_{i}^{*} X_{i} B_{i}\right) \leq \frac{n^{r-1}}{2}\left\|\sum_{i=1}^{n}\left(\left[A_{i}^{*} g^{2}\left(\left|X_{i}^{*}\right|\right) A_{i}\right]^{r}+\left[B_{i}^{*} f^{2}\left(\left|X_{i}\right|\right) B_{i}\right]^{r}\right)\right\|
$$


for all $r \geq 1$.

In the special case, where $f(t)=t^{k}$ and $g(t)=t^{1-k}, \alpha \in(0,1)$, they proved that:

$$
w^{r}\left(\sum_{i=1}^{n} A_{i}^{*} X_{i} B_{i}\right) \leq \frac{n^{r-1}}{2}\left\|\sum_{i=1}^{n}\left(\left[A_{i}^{*}\left|X_{i}^{*}\right|^{2(1-k)} A_{i}\right]^{r}+\left[B_{i}^{*}\left|X_{i}\right|^{2 k} B_{i}\right]^{r}\right)\right\|
$$

In particular, they proved the following inequalities:

1)

$$
w\left(\sum_{i=1}^{n} A_{i}^{*} X_{i} B_{i}\right) \leq \frac{1}{2}\left\|\sum_{i=1}^{n}\left(A_{i}^{*}\left|X_{i}^{*}\right| A_{i}+B_{i}^{*}\left|X_{i}\right| B_{i}\right)\right\|
$$

2)

$$
w^{r}\left(\sum_{i=1}^{n} X_{i}\right) \leq \frac{n^{r-1}}{2}\left\|\sum_{i=1}^{n}\left(\left|X_{i}^{*}\right|^{2 r(1-\kappa)}+\left|X_{i}\right|^{2 r k}\right)\right\|
$$

3)

$$
w^{r}\left(\sum_{i=1}^{n} X_{i}\right) \leq \frac{n^{r-1}}{2}\left\|\sum_{i=1}^{n}\left(\left|X_{i}^{*}\right|^{r}+\left|X_{i}\right|^{r}\right)\right\|
$$

4)

$$
w^{r}(X) \leq \frac{1}{2}\left\|\left(|X|^{r}+\left|X^{*}\right|^{r}\right)\right\|
$$

The main purpose of this paper is to give considerable improvements of the inequalities (7), (8), (9), (10), (11), and (12). In order to achieve our goal, we need the following three lemmas which are essential in our analysis.

The first lemma was proved in [10].

Lemma 1 If $a, b \geq 0$ and $0 \leq v \leq 1$, then:

$$
a^{v} b^{(1-v)}+k(\sqrt{a}-\sqrt{b})^{2} \leq v a+(1-v) b
$$

where $k=\min \{v, 1-v\}$.

If $v=\frac{1}{2}$, the inequality (13) becomes an equality where

$$
\sqrt{a b}=\frac{a+b}{2}-\frac{(\sqrt{a}-\sqrt{b})^{2}}{2}
$$

The second lemma follows from the spectral theorem for positive operators and Jensen's inequality (see [11]).

Lemma 2 Let $T \in B(H), T \geq 0$ and $x \in H$ such that $\|x\| \leq 1$. Then:

1) $\langle T x, x\rangle^{r} \leq\left\langle T^{r} x, x\right\rangle$ for $r \geq 1$.

2) $\left\langle T^{r} x, x\right\rangle \leq\langle T x, x\rangle^{r}$ for $0<r \leq 1$.

The third lemma was proved in [11]

Lemma 3 Let $T \in B(H)$ and $x, y \in H$ be any vectors. If $f, g$ are nonnegative continuous functions on $[0, \infty)$ which are satisfying the relation $f(t) g(t)=t \quad(t \in[0, \infty))$, then: 


$$
|\langle T x, y\rangle|^{2} \leq\langle|T| x, x\rangle\left\langle\left|T^{*}\right| y, y\right\rangle
$$

and more general,

$$
|\langle T x, y\rangle|^{2} \leq\left\langle f^{2}(|T|) x, x\right\rangle\left\langle g^{2}\left(\left|T^{*}\right|\right) y, y\right\rangle
$$

\section{Main Results}

The first result in this paper is numerical radius inequality which is sharper than the inequality (7).

Theorem 3.1 Let $A_{i}, B_{i}, X_{i} \in B(H), i=1,2, \cdots, n$, and $f, g$ be nonnegative continuous functions on $[0, \infty)$ satisfying the relation $f(t) g(t)=t$ $(t \in[0, \infty))$. Then:

$$
w^{r}\left(\sum_{i=1}^{n} A_{i}^{*} X_{i} B_{i}\right) \leq \frac{n^{r-1}}{2}\left\|\sum_{i=1}^{n}\left[A_{i}^{*} g^{2}\left(\left|X_{i}^{*}\right|\right) A_{i}\right]^{r}+\left[B_{i}^{*} f^{2}\left(\left|X_{i}\right|\right) B_{i}\right]^{r}\right\|-\alpha
$$

where

$$
\alpha=\sup _{\|x\|=1} \frac{n^{r-1}}{2} \sum_{i=1}^{n}\left(\left\langle\left[A_{i}^{*} g^{2}\left(\left|X_{i}^{*}\right|\right) A_{i}\right]^{r} x, x\right\rangle^{1 / 2}-\left\langle\left[B_{i}^{*} f^{2}\left(\left|X_{i}\right|\right) B_{i}\right]^{r} x, x\right\rangle^{1 / 2}\right)^{2}
$$

\section{Proof.}

$$
\begin{aligned}
& \left|\left\langle\sum_{i=1}^{n}\left(A_{i}^{*} X_{i} B_{i}\right) x, x\right\rangle\right|^{r} \\
= & \left|\sum_{i=1}^{n}\left\langle\left(A_{i}^{*} X_{i} B_{i}\right) x, x\right\rangle\right|^{r} \\
\leq & \left(\sum_{i=1}^{n}\left|\left\langle\left(A_{i}^{*} X_{i} B_{i}\right) x, x\right\rangle\right|\right)^{r} \\
= & \left(\sum_{i=1}^{n}\left|\left\langle X_{i} B_{i} x, A_{i} x\right\rangle\right|\right)^{r} \\
\leq & \left(\sum_{i=1}^{n}\left\langle f^{2}\left(\left|X_{i}\right|\right) B_{i} x, B_{i} x\right\rangle^{1 / 2}\left\langle g^{2}\left(\left|X_{i}^{*}\right|\right) A_{i} x, A_{i} x\right\rangle^{1 / 2}\right)^{r} \\
\leq & n^{r-1} \sum_{i=1}^{n}\left\langle B_{i}^{*} f^{2}\left(\left|X_{i}\right|\right) B_{i} x, x\right\rangle^{r / 2}\left\langle A_{i}^{*} g^{2}\left(\left|X_{i}^{*}\right|\right) A_{i} x, x\right\rangle^{r / 2} \\
\leq & n^{r-1} \sum_{i=1}^{n}\left\langle\left(B_{i}^{*} f^{2}\left(\left|X_{i}\right|\right) B_{i}\right)^{r} x, x\right\rangle^{1 / 2}\left\langle\left(A_{i}^{*} g^{2}\left(\left|X_{i}^{*}\right|\right) A_{i}\right)^{r} x, x\right\rangle^{1 / 2} \\
= & \frac{n^{r-1}}{2}\left[\sum_{i=1}^{n}\left\langle\left(B_{i}^{*} f^{2}\left(\left|X_{i}\right|\right) B_{i}\right)^{r} x, x\right\rangle+\left\langle\left(A_{i}^{*} g^{2}\left(\left|X_{i}^{*}\right|\right) A_{i}\right)^{r} x, x\right\rangle\right] \\
& -\frac{n^{r-1}}{2}\left[\sum_{i=1}^{n}\left(\left\langle\left(B_{i}^{*} f^{2}\left(\left|X_{i}\right|\right) B_{i}\right)^{r} x, x\right\rangle^{1 / 2}-\left\langle\left(A_{i}^{*} g^{2}\left(\left|X_{i}^{*}\right|\right) A_{i}\right)^{r} x, x\right\rangle^{1 / 2}\right)^{2}\right]
\end{aligned}
$$

Taking the supremum over all unit vectors $x \in H$, we get

$$
\begin{aligned}
& w^{r}\left(\sum_{i=1}^{n} A_{i}^{*} X_{i} B_{i}\right) \\
& \leq \frac{n^{r-1}}{2}\left\|\sum_{i=1}^{n}\left[A_{i}^{*} g^{2}\left(\left|X_{i}^{*}\right|\right) A_{i}\right]^{r}+\left[B_{i}^{*} f^{2}\left(\left|X_{i}\right|\right) B_{i}\right]^{r}\right\|
\end{aligned}
$$




$$
\begin{aligned}
& -\sup _{\|x\|=1} \frac{n^{r-1}}{2} \sum_{i=1}^{n}\left(\left\langle\left[A_{i}^{*} g^{2}\left(\left|X_{i}^{*}\right|\right) A_{i}\right]^{r} x, x\right\rangle^{1 / 2}-\left\langle\left[B_{i}^{*} f^{2}\left(\left|X_{i}\right|\right) B_{i}\right]^{r} x, x\right\rangle^{1 / 2}\right)^{2} \\
& =\frac{n^{r-1}}{2}\left\|\sum_{i=1}^{n}\left[A_{i}^{*} g^{2}\left(\left|X_{i}^{*}\right|\right) A_{i}\right]^{r}+\left[B_{i}^{*} f^{2}\left(\left|X_{i}\right|\right) B_{i}\right]^{r}\right\|-\alpha .
\end{aligned}
$$

Remark 1 In view of the inequalities (7) and (17), it clears that the inequality (17) is sharper than the inequality (7).

As special case of the inequality (17), let $f(t)=t^{\kappa}$ and $g(t)=t^{1-\kappa}$, $\kappa \in(0,1)$, we will get the following inequality which is sharper than the inequality (8).

Corollary 4 Let $A_{i}, B_{i}, X_{i} \in B(H), \quad i=1,2, \cdots, n, \quad r \geq 1$, and $0<\kappa<1$. Then:

$$
w^{r}\left(\sum_{i=1}^{n} A_{i}^{*} X_{i} B_{i}\right) \leq \frac{n^{r-1}}{2}\left\|\sum_{i=1}^{n}\left(\left[A_{i}^{*}\left|X_{i}^{*}\right|^{2(1-\kappa)} A_{i}\right]^{r}+\left[B_{i}^{*}\left|X_{i}\right|^{2 \kappa} B_{i}\right]^{r}\right)\right\|-\beta
$$

where

$$
\beta=\sup _{\|x\|=1} \frac{n^{r-1}}{2} \sum_{i=1}^{n}\left(\left\langle\left[A_{i}^{*}\left|X_{i}^{*}\right|^{2(1-\kappa)} A_{i}\right]^{r} x, x\right\rangle^{1 / 2}-\left\langle\left[B_{i}^{*}\left|X_{i}\right|^{2 \kappa} B_{i}\right]^{r} x, x\right\rangle^{1 / 2}\right)^{2}
$$

In particular, if $r=1, \alpha=\frac{1}{2}$ we get the following inequality which is charper than the inequality (9),

$$
w\left(\sum_{i=1}^{n} A_{i}^{*} X_{i} B_{i}\right) \leq \frac{1}{2}\left\|\sum_{i=1}^{n}\left(A_{i}^{*}\left|X_{i}^{*}\right| A_{i}+B_{i}^{*}\left|X_{i}\right| B_{i}\right)\right\|-\gamma
$$

where

$$
\gamma=\sup _{\|x\|=1} \frac{1}{2} \sum_{i=1}^{n}\left(\left\langle\left[A_{i}^{*}\left|X_{i}^{*}\right| A_{i}\right] x, x\right\rangle^{1 / 2}-\left\langle\left[B_{i}^{*}\left|X_{i}\right| B_{i}\right] x, x\right\rangle^{1 / 2}\right)^{2}
$$

By letting $A_{i}=B_{i}=0$ in the inequality (19), we obtain the following inequality which is sharper than the inequality (10).

Corollary 5 Let $X_{i} \in B(H), i=1,2, \cdots, n, \quad r \geq 1$, and $0<\kappa<1$. Then:

$$
w^{r}\left(\sum_{i=1}^{n} X_{i}\right) \leq \frac{n^{r-1}}{2}\left\|\sum_{i=1}^{n}\left(\left|X_{i}^{*}\right|^{2 r(1-\kappa)}+\left|X_{i}\right|^{2 r k}\right)\right\|-\eta
$$

where

$$
\eta=\sup _{\|x\|=1} \frac{n^{r-1}}{2} \sum_{i=1}^{n}\left(\left\langle\left. X_{i}^{*}\right|^{2 r(1-\kappa)} x, x\right\rangle^{1 / 2}-\left\langle\left|X_{i}\right|^{2 r \kappa} x, x\right\rangle^{1 / 2}\right)^{2}
$$

Letting $\kappa=\frac{1}{2}$ in the inequality (23), we obtain the following inequality which is sharper than the inequality (11).

Corollary 6 Let $X_{i} \in B(H), i=1,2, \cdots, n$, and $r \geq 1$. Then:

$$
w^{r}\left(\sum_{i=1}^{n} X_{i}\right) \leq \frac{n^{r-1}}{2}\left\|\sum_{i=1}^{n}\left(\left|X_{i}^{*}\right|^{r}+\left|X_{i}\right|^{r}\right)\right\|-\zeta
$$


where

$$
\zeta=\sup _{\|x\|=1} \frac{n^{r-1}}{2} \sum_{i=1}^{n}\left(\left\langle\left|X_{i}^{*}\right|^{r} x, x\right\rangle^{1 / 2}-\left\langle\left|X_{i}\right|^{r} x, x\right\rangle^{1 / 2}\right)^{2}
$$

In the inequality (24), replacing $X_{2}, X_{3}, \cdots, X_{n}$ by 0 , we have the following inequality which is sharper than the inequality (12).

Corollary 7 Let $X \in B(H), r \geq 1$. Then:

$$
w^{r}(X) \leq \frac{1}{2}\left\|\left(|X|^{r}+\left|X^{*}\right|^{r}\right)\right\|-\xi
$$

where

$$
\xi=\sup _{\|x\|=1} \frac{1}{2}\left(\left\langle\left|X^{*}\right|^{r} x, x\right\rangle^{1 / 2}-\left\langle|X|^{r} x, x\right\rangle^{1 / 2}\right)^{2}
$$

Now, we will prove the following inequality which is another version of the inequality (6).

Theorem 3.2 Let $A, B \in B(H)$. Then:

$$
w\left(\left[\begin{array}{cc}
0 & A \\
B & 0
\end{array}\right]\right) \leq \frac{\|A\|+\|B\|}{2}
$$

Proof. Let $U=\frac{1}{\sqrt{2}}\left[\begin{array}{cc}I & -I \\ I & I\end{array}\right]$, then $U$ is unitary, and

$$
\begin{aligned}
w\left(\left[\begin{array}{ll}
0 & A \\
B & 0
\end{array}\right]\right)=w\left(U^{*}\right. & {\left.\left[\begin{array}{ll}
0 & A \\
B & 0
\end{array}\right] U\right) \quad((\text { by Equation (3)) }} \\
& =\frac{1}{2} w\left(\left[\begin{array}{cc}
-B-A & -B+A \\
B-A & B+A
\end{array}\right]\right) \\
& =\frac{1}{2} w\left(\left[\begin{array}{ll}
-A & A \\
-A & A
\end{array}\right]+\left[\begin{array}{cc}
-B & -B \\
B & B
\end{array}\right]\right) \\
& \leq \frac{1}{2}\left[w\left(\left[\begin{array}{ll}
-A & A \\
-A & A
\end{array}\right]\right)+w\left(\left[\begin{array}{cc}
-B & -B \\
B & B
\end{array}\right]\right)\right] \\
& =\frac{\|A\|+\|B\|}{2}
\end{aligned}
$$

since $\left(\left[\begin{array}{cc}-A & A \\ -A & A\end{array}\right]^{2}=0\right.$, so $\left.\left(\left[\begin{array}{ll}-A & A \\ -A & A\end{array}\right]\right)=\frac{\left\|\left[\begin{array}{ll}-A & A \\ -A & A\end{array}\right]\right\|}{2}=\|A\|\right)$,

and $\left(\left[\begin{array}{cc}-B & -B \\ B & B\end{array}\right]^{2}=0\right.$, so $\left.w\left(\left[\begin{array}{cc}-B & -B \\ B & B\end{array}\right]\right)=\frac{\left\|\left[\begin{array}{cc}-B & -B \\ B & B\end{array}\right]\right\|}{2}=\|B\|\right)$.

Chaining the inequality (27) with the inequality (4) yields the following inequality.

Corollary 8 Let $A, B \in B(H)$. Then:

$$
w\left(\left[\begin{array}{cc}
0 & A \\
B & 0
\end{array}\right]\right) \leq w(A)+w(B)-\frac{\|\operatorname{Re} A\|-\|\operatorname{Im} A\| \mid}{2}-\frac{\|\operatorname{Re} B\|-\|\operatorname{Im} B\|}{2}
$$


Proof. In Theorem 3.2, apply the inequality (4) on the right side, we get the result.

Chaining the inequality (27) with the inequality (5) yields the following inequality.

Corollary 9 Let $A, B \in B(H)$. Then:

$$
\begin{gathered}
w\left(\left[\begin{array}{cc}
0 & A \\
B & 0
\end{array}\right]\right) \leq \\
-\frac{\left|\|\operatorname{Re} B\|-\frac{\|B\|}{2}\right|}{4}-\frac{\left|\|\operatorname{Im} B\|-\frac{\|B\|}{2}\right|}{4}
\end{gathered}
$$

Proof. In Theorem 3.2, apply the inequality (5) on the right side, we get the result.

\section{Conflicts of Interest}

The authors declare no conflicts of interest regarding the publication of this paper.

\section{References}

[1] Gustafson, K.E. and Rao, D.K.M. (1997) Numerical Range, the Field of Values of Linear Operators and Matrices. Springer, New York.

https://doi.org/10.1007/978-1-4613-8498-4_1

[2] Halmos, P.R. (1982) A Hilbert Space Problem Book. 2nd Edition, Springer, New York. https://doi.org/10.1007/978-1-4684-9330-6

[3] Hirzallah, O., Kittaneh, F. and Shebrawi, K. (2011) Numerical Radius Inequalities for Commutators of Hilbert Space Operators. Numerical Functional Analysis and Optimization, 32, 739-749. https://doi.org/10.1080/01630563.2011.580875

[4] Kittaneh, F. (2005) Numerical Radius Inequalities for Hilbert Space Operators. Studia Mathematica, 168, 73-80. https://doi.org/10.4064/sm168-1-5

[5] Kittaneh, F. (2003) A Numerical Radius Inequality and an Estimate for the Numerical Radius of the Frobenius Companion Matrix. Studia Mathematica, 158, 11-17. https://doi.org/10.4064/sm158-1-2

[6] Sheikhhosseini, A., Moslehian, M.S. and Shebrawi, Kh. (2017) Inequalities for Generalized Euclidean Operator Radius via Youngs Inequality. Journal of Mathematical Analysis and Applications, 445, 1516-1529. https://doi.org/10.1016/j.jmaa.2016.03.079

[7] Yamazaki, T. (2007) On Upper and Lower Bounds of the Numerical Radius and an Equality Condition. Studia Mathematica, 178, 83-89.

https://doi.org/10.4064/sm178-1-5

[8] Hirzallah, O., Kittaneh, F. and Shebrawi, K. (2011) Numerical Radius Inequalities for Certain $2 \times 2$ Operator Matrices. Integral Equations and Operator Theory, 71, 129-147. https://doi.org/10.1007/s00020-011-1893-0

[9] Shebrawi, K. and Albadawi, H. (2009) Numerical Radius and Operator Norm Inequalities. Journal of Inequalities and Applications, 2009, Article ID: 492154. https://doi.org/10.1155/2009/492154 
[10] Kittaneh, F. and Manasrah, Y. (2010) Improved Young and Heinz Inequalities for Matrices. Journal of Mathematical Analysis and Applications, 361, 262-269. https://doi.org/10.1016/j.jmaa.2009.08.059

[11] Kittaneh, F. (1988) Notes on Some Inequalities for Hilbert Space Operators. Publications of the Research Institute for Mathematical Sciences, 24, 283-293. https://doi.org/10.2977/prims/1195175202 